\title{
Photonic multilayer structure of Begonia chloroplasts enhances photosynthetic efficiency
}

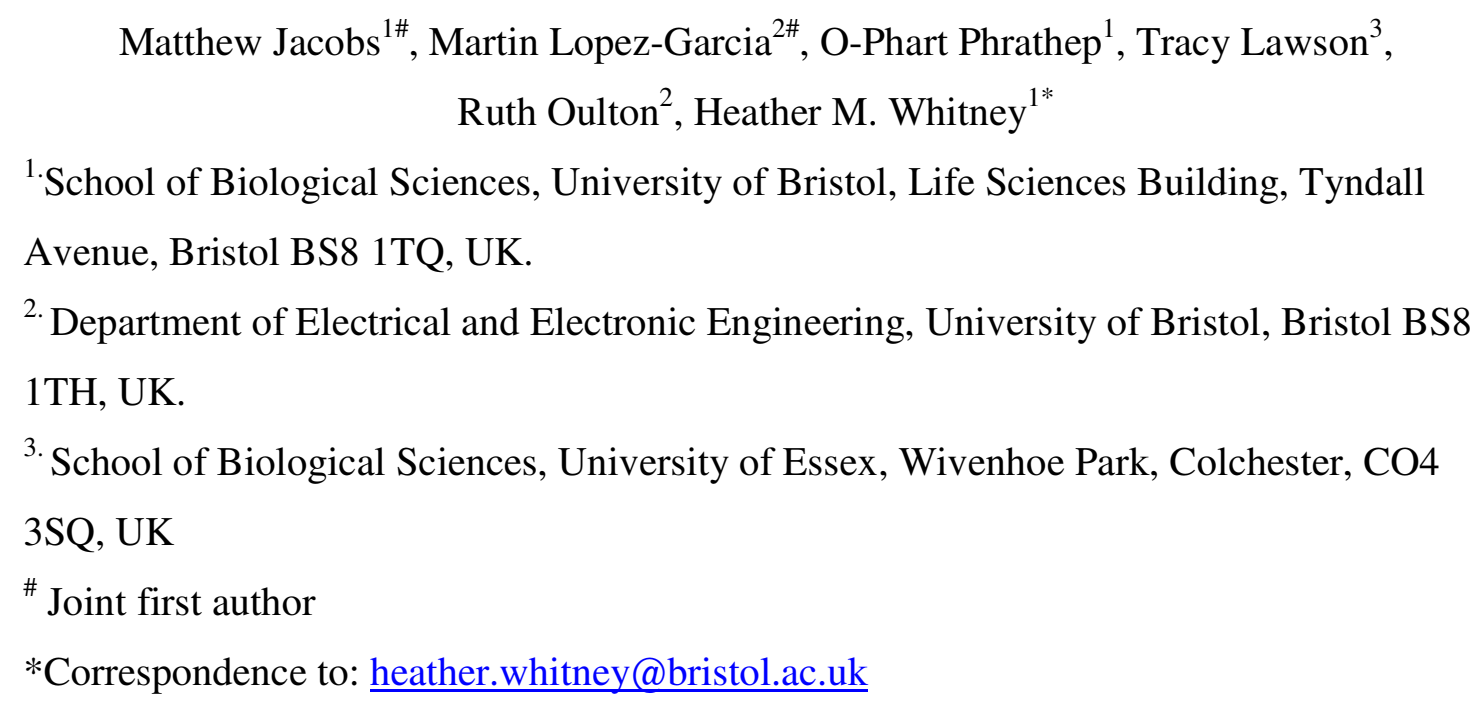

Enhanced light-harvesting is an area of interest for optimising both natural photosynthesis and artificial solar energy capture ${ }^{1,2}$. While iridescence has been shown to exist widely and in diverse forms in plants and other photosynthetic organisms and symbioses ${ }^{3,4}$, there has yet to be any direct link demonstrated between iridescence and photosynthesis. Here we show that epidermal chloroplasts, also known as iridoplasts, in shade-dwelling species of Begonia ${ }^{5}$, notable for their brilliant blue iridescence, have a photonic crystal structure formed from a periodic arrangement of the light-absorbing thylakoid tissue itself. This structure enhances photosynthesis in two ways: by increasing light capture at the predominantly green wavelengths available in shade conditions, and by directly enhancing quantum yield by $10-15 \%$ under low light conditions. These findings together imply that the iridoplast is a highly modified chloroplast structure adapted to make best use of the extremely low light conditions in the tropical forest understory in which it is found ${ }^{5,6}$. A phylogenetically diverse range of shade-dwelling plant species have been found to produce similarly structured chloroplasts ${ }^{7-9}$, suggesting that the ability to produce chloroplasts whose 
membranes are organized as a multilayer with photonic properties may be widespread. In fact, given the well-established diversity and plasticity of chloroplasts ${ }^{10,11}$, our results imply that photonic effects may be important even in plants that do not show any obvious signs of iridescence to the naked eye but where a highly ordered chloroplast structure may present a clear blue reflectance at the microscale. Chloroplasts are generally thought of as purely photochemical; we suggest that one should also think of them as a photonic structure with a complex interplay between control of light propagation, light capture, and photochemistry.

Photonic crystals are periodic nanoscale structures which interact with light, resulting in a number of optical phenomena. In artificial systems, these photonic properties have been investigated for their light harvesting properties as they can strongly enhance the performance of devices for solar energy production, through either light-trapping ${ }^{1}$ or slow light mechanisms ${ }^{2}$. However, one group of photonic crystals that have repeatedly been associated with light harvesting roles including optimised light distribution within photosynthetic tissues ${ }^{3}$, but which have yet to be directly linked to enhanced light harvesting, are natural biological photonic crystals. Photonic structures are widespread in nature, where they are typically associated with structural colour ${ }^{4,12,13}$. Although more extensively researched in animals, photonic effects have further implications in plants given the importance of light manipulation for photosynthesis ${ }^{4}$. A striking example of structural colour in plants is presented by the iridescent blue leaves observed in a diverse range of tropical plant species adapted to deep forest shade conditions, however little direct evidence of the function of this structural colour has been presented ${ }^{14,15}$. 
Blue iridescent leaf colouration has been reported in the genus Begonia and explored in a single species, B.pavonina ${ }^{16}$. Unusual plastids containing highly ordered internal structures termed iridoplasts were observed in the adaxial epidermis and proposed as the source of the blue colouration $^{5}$. However, it has never been demonstrated whether iridoplasts are indeed responsible for this blue structural colouration, or what biological function it signifies.

To investigate Begonia leaf iridescence directly, we imaged leaves of B.grandis $\times$ pavonina. This hybrid was generated and used for the majority of work as it displays the intense iridescence typical of B.pavonina whilst maintaining the more vigorous growth habit typical of B.grandis. Figure 1a shows the vivid iridescence observed in the mature leaves of this hybrid. Using reflected light microscopy, we imaged (Fig. 1b) and measured spectra (Fig. 1d) from single iridoplasts in vivo. Both confirm that iridoplasts show a strong blue peak in reflectance observed at central wavelength $\lambda_{\mathrm{c}} \approx 470 \mathrm{~nm}$ with a spectral width of $\sim 60 \mathrm{~nm}$. Both TEM (Supplementary Fig. S1) and freeze-fracture cryo-SEM (Fig. 1c) clearly show regularly spaced grana. Iridoplasts were observed in all adaxial epidermal cells of a phylogenetically diverse selection of Begonia species, including in leaves where iridescence was not visible to the naked eye. TEM further confirmed the presence of the highly ordered iridoplasts in these species (Supplementary Fig. S2). Despite some variation in spectral width, both the observed colour and corresponding $\lambda_{\mathrm{c}}$ is similar for all iridoplasts in all species measured to date, with no observed reflectance out of the blue-turquoise region $\left(450<\lambda_{c}<500 \mathrm{~nm}\right)$. We also confirmed that both the iridoplasts and archetypal mesophyll chloroplasts ('chloroplast' from herein) show similar variation in autofluorescence (Supplementary Fig. S3) indicating that iridoplasts may show a photosynthetic function. 
To explore the possible biological function, we examined the micro and nanoscopic morphology

of the iridoplast in quantitative detail to obtain input data for an optical model of the absorption and reflectance of the structure. Chloroplast structure is well established (Supplementary Fig. S7, ${ }^{10,17}$ ) however in iridoplasts only a few thylakoids (three in Fig.2a) are stacked into grana of $\sim 40 \mathrm{~nm}$ thickness, that are themselves regularly spaced by $d_{\mathrm{s}} \sim 100 \mathrm{~nm}$. Ultrastructural analysis revealed that each granum is formed from the same number of thylakoids $\left(N_{m}\right)$ throughout the iridoplast (Supplementary Fig.4). The average number of thylakoids per granum can vary with $N_{\mathrm{m}}=3.3 \pm 0.8$ between iridoplasts. Fast Fourier Transform (FFT) imaging (Supplementary Fig. S5) allows an accurate estimate of the total period between grana to be $\Lambda=$ $170 \pm 20 \mathrm{~nm}$. The thickness of the thylakoid membrane $(M / 2)$ and the lumen $(L)$ were estimated from the TEM to be $M=6.68 \pm 0.76 \mathrm{~nm}$ and $L=7.52 \pm 0.82 \mathrm{~nm}$ respectively.

These data were used as input into an optical transfer matrix method (TMM) model (see Methods) that calculates electric field intensity and reflectance for multiple periodic layers of varying refractive index (Fig. 2a). The reflectance spectra of single iridoplasts were analysed and compared with that predicted by our model (Fig. 2b). A high level of replication for the spectral shape of the reflectance is obtained with values of $d_{\mathrm{s}}=125,115 \mathrm{~nm}$. To establish if this model predicts the formation of a photonic stopband within the observed thickness parameters we modelled a variation of $d_{\mathrm{s}}$ over a wide range of values from $d_{\mathrm{s}}=0$ to $250 \mathrm{~nm}$ for a constant number of grana $(N=8)$. As shown in Fig. $2 c$, no strong reflectance at any wavelength is observed when $d_{\mathrm{s}}=0$, mimicking chloroplasts with partition gaps between grana of a few nanometers ${ }^{18}$. However in the range $100<d_{\mathrm{s}}<160 \mathrm{~nm}$ a strong reflectance peak appears at short 
wavelength for small $d_{\mathrm{s}}(\lambda \approx 430 \mathrm{~nm})$ and at long wavelengths for long $\mathrm{d}_{\mathrm{s}}(\lambda \approx 560 \mathrm{~nm})$. This is a clear indication that the chlorophyll pigments in the thylakoid membranes are capable not only of absorption but also sufficient modification of the real part of the refractive index to produce a photonic stop-band. The data also indicate the approximate range of wavelengths over which one might see peak reflectance $(430-560 \mathrm{~nm})$. Measurement of 20 iridoplasts (Supplementary Fig. S6) confirms a range of peak reflectance from $\sim 435-500 \mathrm{~nm}$, towards the blue end of the calculated range. Finally, we also performed angular reflectance measurements to evaluate the angular dependence of the iridoplast optical properties. As expected from a multilayer structure we obtained a blueshift of the reflectance peak as the angle of incidence is increased (Supplementary Fig. S6). Comparison with calculations using the optical model described before also shows a good agreement.

This model was used to evaluate the effect of iridoplast photonic structure on absorption at the thylakoid membranes. Photonic crystals can strongly enhance or reduce absorption depending on absorber spatial position and spectral response. The mechanism by which light-matter interaction leads to enhanced absorptance when the spacing period is close to the wavelength of light in the structure is known as "slow light". This phenomenon arises from the reduction of the group velocity of the light propagating through the photonic crystal for those wavelengths within the photonic band edge ${ }^{19}$, in our case $\lambda \approx 440 \mathrm{~nm}$ and $\lambda \approx 520 \mathrm{~nm}$ (Fig. 2). When light propagates through a photonic crystal at, or close to the stop-band, interference of forward and backward propagating light leads to the formation of a standing wave with nodes (low $|E|^{2}$ ) and antinodes (high $|E|^{2}$ ) at specific locations. Interestingly it is for the longer wavelength edge of the photonic band where the electric field is concentrated in the high refractive index layers, in our case, the thylakoid membranes. The absorptance will then be enhanced for those wavelengths for which 
the slow light phenomena occurs simultaneously with the appropriate positioning of the photosynthetic membranes within the iridoplast. In general terms it is established that while absorption is reduced for those wavelengths within the photonic band gap (usually shown as strong reflectance in natural photonics) it can be strongly enhanced at the band gap edges ${ }^{19}$. We calculated the absorptance of the organelles as $A^{I r}(\lambda)=1-R(\lambda)-T(\lambda)^{2}$, where $R$ and $T$ are the reflectance and transmittance of the multilayer at wavelength $\lambda$ respectively. Using the previous structural parameters we compared $A^{I r}$ with the absorptance of the same structure $\left(A^{C h l o}\right)$ without partition gap between grana $\left(d_{\mathrm{s}}=0\right.$, i.e. similar to an unmodified chloroplast $\left.{ }^{20}\right)$, defining the absorptance enhancement factor as the ratio $\gamma(\lambda)=A^{I r}(\lambda) / A^{C h l o}(\lambda)$. One obtains an absorptance enhancement $(\gamma>1)$ for all wavelengths in the green-red spectral range $(500<\lambda<700 \mathrm{~nm})$ and a reduction $(\gamma<1)$ in the blue $(\lambda<500 \mathrm{~nm})$ as seen in Fig. 3a. The cut-off wavelength for $\gamma>1$ will depend on $d_{s}$, with two values for $d_{\mathrm{s}}$ plotted $(125 \mathrm{~nm}, 115 \mathrm{~nm})$. In fact, single wavelength analysis unveils maximum enhancements (reductions) of $\gamma=1.27(0.73)$ for $\lambda=523(466) \mathrm{nm}$ and $d_{\mathrm{s}}=125 \mathrm{~nm}$. Fig. $3 \mathrm{~b}$ shows a calculation of the spatial distribution of the light intensity $|E|^{2}$ across the grana and stroma. Fig. $3 \mathrm{~b}$ also shows the position of the grana with respect to the antinodes for different wavelengths. For blue light $(460 \mathrm{~nm})$, the grana are located at the nodes, while for $530 \mathrm{~nm}$ (green) the grana are located at antinodes. This explains intuitively why the calculations in Fig. 3a show relatively more absorptance in the green and less in the blue spectral regions. The angular dependency of the iridoplast optical properties could also influence the absorptance. Therefore, we studied the angular dependence of $\gamma$ (Fig. 3c). As can be observed, the region where $\gamma>1$ (absorptance enhancement) extends to incident angles $\theta<30^{\circ}$. Interestingly the angular dependence for $\gamma$ is not very strong showing an almost constant maximum value $\gamma \approx 1.2$ for $500<\lambda<550 \mathrm{~nm}$. This would support our hypothesis of the blue 
coloration being a by-product of the light harvesting functionality of the iridoplast given that the reflectance peak is blueshifted for large incident angles (Supplementary Fig. S6).

Iridoplasts therefore both reflect blue light and show enhanced absorption in the green-red. These Begonia species are found in "extreme" shade conditions. The overhead forest canopy absorbs most of the light, such that the intensity reaching the forest floor can be attenuated by up to $10^{-6}$ $10^{-7}(60-70 \mathrm{~dB})^{6}$. Moreover, the spectral distribution of available light is modified: absorption of the $\sim 460 \mathrm{~nm}$ and $\sim 680 \mathrm{~nm}$ regions by the canopy above results in a modified spectrum remaining for the understory Begonia (Fig. 3a). Enhanced absorption in the green region of the spectrum may therefore be a way to scavenge residual light. The reduction in relative enhancement factor for the blue (460nm) would not be a disadvantage given the very low levels of these wavelengths available, and may even aid photo-protection ${ }^{14}$.

Direct insight into the impact of these modifications on overall photosynthetic efficiency may be gained by using chlorophyll fluorescence imaging of iridoplasts and chloroplasts in the Begonia leaf tissue. Chlorophyll fluorescence imaging has become a standard technique to assess photosynthetic parameters in a range of organisms. While it gives no information on how absorption affects the overall efficiency of photosynthesis, this technique provides an estimate of the efficiency (quantum yield) with which absorbed light can be utilized for electron transport and photosynthesis ${ }^{21,22}$.

Figure 4 shows that maximum quantum efficiency of PSII photochemistry $\left(\mathrm{F}_{\mathrm{v}} / \mathrm{F}_{\mathrm{m}}\right)$ values from iridoplasts were significantly $(p<0.05)$ and consistently $5-10 \%$ higher than those of 
chloroplasts, both in cross sections and in peels comprising adaxial epidermis and mesophyll (Supplementary Fig. S8). This is surprising given that lower efficiency has been observed in other epidermal chloroplasts ${ }^{23}$, and thus it appears that the photonic structure may aid in postabsorptive efficiency. Consistent with previous studies of epidermal chloroplasts however ${ }^{22}$, iridoplasts show reduced efficiency in higher light conditions (Supplementary Fig. S9), indicating limitation by electron transport downstream of PSII. Shade-adapted chloroplasts typically show an increase in appressed: non-appressed thylakoids (as seen in iridoplasts) and an associated increase in the amount of PSII antenna complexes ${ }^{24}$. Interestingly, these adaptations have been shown to reduce quantum yield ${ }^{25}$ and so it may be that the iridoplast photonic structure compensates for this evolutionary trade-off.

The combination of these results suggests that iridoplasts are particularly adapted for low light conditions, where other plants would struggle to grow. Under such conditions the observed reduction in efficiency at higher light levels would not pose a disadvantage to the plant and the potential trade-off of less efficient electron transport would have fewer photodamage implications. While the enhancement of whole plant photosynthesis by the presence of iridoplasts may be marginal, in extreme conditions strong selective pressures would be sufficient to maintain the presence of these structures. This hypothesis is further supported by description of chloroplast structures similar to those found in Begonia iridoplasts in a phylogenetically diverse selection of plants from similar deep shade environments, although to our knowledge few of them are described as iridescent and none have yet been modelled as a photonic crystal $^{7-9}$. This work provides an initial link between physical photonic modification and photosynthetic quantum yield in chloroplasts, but potentially, iridoplasts are just one, clearly visible example of a wide variety of photonic adaptation in light harvesting complexes to be discovered. 
Methods

Electron microscopy. For transmission electronic microscopy (TEM), leaf tissue was fixed in a

$2.7 \%$ glutaraldehyde solution in $0.1 \mathrm{M}$ sodium cacodylate buffer $(\mathrm{pH} 7.2)$ at room temperature overnight and post-fixed in $1 \%$ osmium tetroxide in $0.1 \mathrm{M}$ sodium cacodylate for 1.5 hours at room temperature. Specimens were dehydrated in an ethanol series before being embedded in LR white resin (Sigma Aldrich) and 70nm sections taken with a diamond knife. Sections were stained with aqueous uranyl acetate and lead citrate and imaged using a Tecnai T12 microscope (FEI). Representative images are taken from 5 technical repeats for Begonia grandis x pavonina. For cryogenic scanning electron microscopy (cryo-SEM) the leaf tissue was plunge-frozen in liquid nitrogen slush and transferred immediately to a Quanta 400 - Scanning Electron Microscope (FEI) for sputter coating with platinum and imaging.

Autofluorescence imaging. Slides were prepared from dark-adapted leaves either as cross sections or peels containing both adaxial epidermis and palisade mesophyll. Specimens were kept in incubation buffer $\left(50 \mathrm{mM} \mathrm{KCl}, 10 \mathrm{mM}\right.$ PIPES, $\mathrm{pH} 6.8,{ }^{26}$ under a coverslip and further dark adapted for at least 15 mins prior to imaging. Fluorescence images were taken with a Fluorescence Kinetic Microscope controlled with FluorCam7 software (both Photon Systems Instruments). Plastids were selected from fluorescence images using thresholding in FluorCam7 and exported data were analysed with Microsoft Excel 2013 and R (version 3.2.3). Values were calculated by averaging data from all plastids in each replicate. Data in Fig. $4 \mathrm{~b}$ are the means of 18 replicates for mesophyll chloroplasts and iridoplasts and 4 replicates for guard cell 
chloroplasts, from 10 technical repeats. Measurements were discarded if an actinic effect of measuring light was detected, indicated by a non-zero gradient during determination of F0. Data in Supplementary Fig. S9 are the means of at least 6 replicates. Data were analyzed using oneway ANOVA and pairwise t-tests (with Bonferroni correction since the number of comparisons is low). For the data in Fig. 4, one-way ANOVA gave $\mathrm{p}=9.39 \mathrm{e}-07$. Pairwise t-tests: Iridoplasts:Mesophyll Chloroplasts $\mathrm{p}=0.02$; Guard cell chloroplasts:Mesophyll Chloroplasts $\mathrm{p}=$ 3.4e-05; Guard cell chloroplasts:Iridoplasts $\mathrm{p}=5.3 \mathrm{e}-07$.

Plant material. Begonia plants were grown in glasshouse conditions supplemented with compact fluorescent lighting (Plug and Grow 125W, 6400K, LBS Horticultural Supplies). Plants were grown under benches, with additional shade provided by $50 \%$ roof shade netting (Rokolene).

Reflectance measurements. High resolution imaging and spectra of iridoplasts were performed in vivo on dark-adapted leaves under a coverslip. For characterization we used a custom-made white light epi-illumination microscope. White light lamp illumination (Thorlabs OSL-1) was collimated and focused on the sample with a high NA (1.4) oil immersion lens (Zeiss PlanApochromat 100x/1.4 Oil M27). The collected light was then focused onto an optical fiber (Thorlabs M92L01) in a confocal configuration. The optical fiber is connected to an Ocean Optics 2000+ spectrometer for spectral analysis. This configuration ensures the capability to select single iridoplasts in our measurements. A modified configuration of the previous setup allowed Fourier Image Spectroscopy ${ }^{27}$ over the whole numerical aperture of the objective lens therefore allowing selection of different angles of collection. All reflectance spectra are normalized against a silver mirror (Thorlabs PF10-03-P01) used as the standard for our measurements. 
Confocal photoluminescence excitation (PLE). PLE measurements (also known as Lambda Square Mapping) were performed on a confocal optical microscope Leica SP8X. Using the confocal capabilities we could distinguish PLE signal from iridoplasts and chloroplasts for the same region of the leaf (Supplementary Fig. S3). Values were calculated by averaging the signal from organelles in the same confocal image. For Extended Data Fig 3a the number of samples for iridoplasts was $n=7$ and $n=10$ for chloroplasts.

Optical model of iridoplasts. For the calculation of the reflectance spectra of the iridoplast we used an in-house implementation of the Transfer Matrix Method (TMM) ${ }^{28}$ for unidimensional photonic crystals. We performed a layer by layer calculation defining each layer of the iridoplast. In these simulations each layer needed two parameters as input: refractive index and thickness.

Note that unlike in standard calculations in quarter wavelength stacks, the photonic structure under study here presents an ultrastructure in which each high refractive index layer (thylakoid membrane) is less than ten nanometers in thickness. This ultrastructure cannot be approximated by a layer several tens of nanometers thick with a given effective refractive index since variations in thylakoid lumenic thickness would not be accounted for in this simplified model. Therefore, the thickness of each layer is defined with nanometric precision in our calculation.

In order to obtain values of those thicknesses, we performed an exhaustive analysis of TEM images to obtain statistics (Extended Data Fig. S4) on the most common values for $M / 2, L$ and $d_{s}$. As described in Fig 2 of the main text, those layers are piled up to form a superstructure where each granum is periodically replicated with a period (A). The granum ultrastructure, that is, the number of thylakoids forming a single granum $\left(N_{m}\right)$, is also obtained from statistical 
analysis of TEM images (Supplementary Fig. S4). The total grana thickness is then defined as $d_{m}=N_{m}(M+L)$.

For the refractive indices used in this model we relied upon well-established data in the literature. The low refractive index layers in the iridoplast are stroma and lumen. They are wellknown to have an aqueous composition and therefore their refractive index is considered real (non-absorbing) and close to water $(1.3<n<1.4)$. The exact values however are difficult to obtain $^{29}$ as well as very variant due to changes in the chemical composition, particularly in the lumen of thylakoids where protein exchange is common ${ }^{18}$. In our case we used values $n_{L}=n_{s}=1.35$ as lumen and stroma are known to have very similar values. For the thylakoid membrane, the presence of pigments (especially chlorophyll) will produce a strong material dispersion. The refractive index for the thylakoid membrane $\left(n_{t}=n+i k\right)$ is complex (the imaginary part, $k$, corresponding to absorption) and varies with wavelength. This is partly due to pigments such as chlorophyll (present in both chloroplasts and iridoplasts, absorbing strongly in the blue ( 420nm) and red (680nm) which causes a change in the real part of the refractive index at those resonant wavelengths. Refractive index values are shown in Supplementary Fig. S3.

In our approach to modelling the iridoplast we defined each layer according to the thickness extracted from ultrastructural analysis. Next we select the refractive index depending on the type of material the layer is formed from. Note that while lumen and stroma will not show material dispersion, the thylakoid membrane refractive index will change strongly with light wavelength. Therefore, in order to define the refractive index of the thylakoid membrane we selected the appropriate complex refractive index for the wavelength of interest as shown in Supplementary Fig. S3. Finally, reflectance was calculated for single wavelengths incident normally to the superstructure and from an aqueous medium with refractive index $n_{i n}=1.33$. 
Note that the inner chemistry and especially the ultrastructure of chloroplasts and iridoplasts changes to adapt to the light environment ${ }^{23}$. Therefore, the reflectance of the iridoplast as calculated with values extracted from TEMs is likely to differ from the actual reflectance measurement given the difficulty of measuring reflectance and TEM of the same iridoplast. The mean values as extracted from TEM (sample size $n=17$ ) images (Extended Data Fig. 4) were $M=6.7 \pm 0.8 \mathrm{~nm}, L=7.5 \pm 0.8 \mathrm{~nm}, d_{s}=94 \pm 16 \mathrm{~nm}$ (sample size $\left.n=70\right)$. Numbers of grana and thylakoids per granum were: $N=7 \pm 1$ and $N_{m}=3 \pm 1$. When these values were introduced into the model a strongly blue shifted and reduced reflectance was obtained. However, after performing a fine tuning of these parameters we determined that for values $M=9 \mathrm{~nm}, L=7.5 \mathrm{~nm}$ and $d_{s}$ in the range 115-125 nm a very good fit was obtained. The other parameters were $N=8$ and $N_{m}=3$. Finally, for those values the model suggests a period $\Lambda=174.5 \mathrm{~nm}$ with $d_{m}=49.5 \mathrm{~nm}$.

Variance on thicknesses. The thicknesses of iridoplasts layering predicted by our model are still well within values reported in literature for different membranes and compartments in chloroplasts $^{29}$ as well as within the errors obtained from our own statistical analysis, except for the case of the thylakoid membrane. The causes of this mismatch are twofold. First, the distances in TEM images are usually underestimated due to shrinkage during the tissue preparation. Therefore the values for $L, M$ and $d_{\mathrm{s}}$ obtained in Supplementary Fig. S4 are likely to be slightly smaller than in vivo. On the other hand, cryo-SEM (Fig. 1c) is known to produce more realistic distances $^{29}$. Interestingly, when an FFT study of cryo-SEM images of a single iridoplast was performed we obtained a period $\Lambda \approx 170 \pm 20 \mathrm{~nm}$ (Supplementary Fig S7). This value is very similar to the period suggested by the model, fitting experimental reflectance measurements. The second cause for differences between experimental and modelling values is that the inspection of the thickness of thylakoid membranes and their relation to the actual structure of the organelles 
in-vivo is very challenging. Thickness of $L$ and $M$ is known to change with light adaptation but also the arrangement of grana ${ }^{10}$. All our morphological analysis was performed in leaves showing strong iridoplast reflectance after dark adaptation. However, iridoplast reflectance appears to be very sensitive to light conditions. Hence, slight differences in conditions during preparation for electron and optical microscopy might also be responsible for the differences in the values of thicknesses between the model and TEM images. Further work is required on the dynamics of thylakoids in iridoplasts but this is beyond the scope of this study.

Calculation of electric field profiles within an iridoplast under illumination. For calculation of field profiles we used a commercial Finite Difference Time Domain (FDTD) tool ${ }^{30}$. We used the same structure and refractive indices used in the TMM implementation. A single wavelength plane wave illumination normal to the multilayer was used as an excitation source and the field was allowed to propagate within the structure for $3 \mathrm{ps}$.

Alternative description of the enhanced absorption. For a simpler structure than the iridoplast, an alternative derivation for the dimensionless enhancement factor $\gamma$ can be obtained ${ }^{19}$ :

$$
\gamma=f \frac{c / n_{l}}{v_{g}}
$$

where $f$ is a dimensionless parameter representing the overlap in position between the structure of the absorbent material of the photonic crystals and the field distribution. $n_{l}$ is the real part of the refractive index of absorbent material (bulk) and $\mathrm{v}_{\mathrm{g}}$ is the group velocity of the light wave. Therefore, a higher field-grana overlap and lower group velocity will both increase $\gamma$ as our model demonstrates for iridoplasts due to the presence of the photonic band edge. The total absorptance enhancement parameter $\left(\gamma_{\text {tot }}\right)$ mentioned in the caption of Fig. 3 is calculated as: 


$$
\gamma_{t o t}=\int^{P A R} \gamma(\lambda) d \lambda
$$

Where the Photosynthetically Active Radiation (PAR) range is $400 \mathrm{~nm}<\lambda<700 \mathrm{~nm}$ and $\gamma$ the dimensionless enhancement factor at a particular wavelength $\lambda$.

Requests for material: Please contact the corresponding author (heather.whitney@bristol.ac.uk) to request any material. The custom code used for implementation of the Transfer Matrix Method was developed as a Matlab script and is available upon request from Martin LopezGarcia (eemlg@bristol.ac.uk).

Acknowledgements: We would like to thank Dr Catherine Kidner, Dr Mark Hughes (Royal Botanic Gardens Edinburgh) and Mr Clive Lundquist (Royal Botanic Gardens Kew) for Begonia species identification and tissue, and Mrs Alanna Kelly for Begonia collection maintenance and horticultural expertise. We thank Stuart Casson and Lucy Sandbach for contributions to pilot studies. We acknowledge the Wolfson Bioimaging Facility (University of Bristol) with thanks to Dominic Alibhai, Judith Mantell and Gini Tilly for assistance with imaging and also thank Dr Silvere Vialet-Chabrand (University of Essex) for assistance with fluorescence microscopy. We also thank Prof Martin J. Cryan, Dr Tom Oliver and Dr. Sara Nunez from the University of Bristol for fruitful discussion on the photonic side of photosynthesis. MJ is funded by a NERC PhD studentship. We are grateful for funding from ERC project number 260920 (to HMW).

Author Contributions: M.J., M.L-G., R.O. and H.W. conceived the experiments, M.J., O.P. and M.L-G. carried out iridoplast optical analysis, M.J., M.L-G. and T.S. carried out the photosynthesis experiments, M.J., O.P. and M.L-G. carried out microscopy, M.L-G. and R.O. designed and ran optical models, M.J., M.L-G., R.O. and H.W. wrote the manuscript, which all authors commented on. 


\section{References}

1. Bermel, P., Luo, C., Zeng, L., Kimerling, L. C. \& Joannopoulos, J. D. Improving thin-film crystalline silicon solar cell efficiencies with photonic crystals. Opt. Express 15, 1698617000 (2007).

2. Mihi, A. \& Miguez, H. Origin of light-harvesting enhancement in colloidal-photoniccrystal-based dye-sensitized solar cells. J. Phys. Chem. B 109, 15968-15976 (2005).

3. Holt, A. L., Vahidinia, S., Gagnon, Y. L., Morse, D. E. \& Sweeney, A. M. Photosymbiotic giant clams are transformers of solar flux. J. R. Soc. Interface 11, 20140678 (2014).

4. Glover, B. J. \& Whitney, H. M. Structural colour and iridescence in plants: the poorly studied relations of pigment colour. Ann. Bot. 105, 505-511 (2010).

5. Gould, K. S. \& Lee, D. W. Physical and ultrastructural basis of blue leaf iridescence in four Malaysian understory plants. Am. J. Bot. 83, 45-50 (1996).

6. Endler, J. A. The color of light in forests and its implications. Ecol. Monogr. 63, 1-27 (1993).

7. Sheue, C., Sarafis, V. \& Kiew, R. Bizonoplast, a unique chloroplast in the epidermal cells of microphylls in the shade plant Selaginella erythropus (Selaginellaceae). Am. J. Bot. 94, 1922-1929 (2007).

8. Graham, R. M., Lee, D. W. \& Norstog, K. Physical and ultrastructural basis of blue leaf iridescence in two neotropical ferns. Am. J. Bot. 80, 198-203 (1993).

9. Nasrulhaq-Boyce, A. \& Duckett, J. Dimorphic epidermal cell chloroplasts in the mesophyll-less leaves of an extreme-shade tropical fern, Teratophyllum rotundifoliatum (R. Bonap.) Holtt.: a light and electron microscope study. New Phytol. 119, 433-444 (1991).

10. Kirchhoff, H. Architectural switches in plant thylakoid membranes. Photosynth. Res. 116, 481-487 (2013).

11. Pribil, M., Labs, M. \& Leister, D. Structure and dynamics of thylakoids in land plants. $J$. Exp. Bot. 65, 1955-1972 (2014).

12. Vukusic, P. \& Sambles, J. R. Photonic structures in biology. Nature 424, 852-855 (2003).

13. Sun, J., Bhushan, B. \& Tong, J. Structural coloration in nature. RSC Adv. 3, 14862-14889 (2013).

14. Thomas, K. R., Kolle, M., Whitney, H. M., Glover, B. J. \& Steiner, U. Function of blue iridescence in tropical understorey plants. J. R. Soc. Interface 7, 1699-1707 (2010).

15. Lee, D. W. Nature's Pallete: the Science of Plant Colour. (The University of Chicago Press, 2007).

16. Gebushuber, C. I. \& Lee, D. W. in Springer Encycl. Nanotechnol. (Bhushan, B.) 17901803 (Springer Netherlands, 2012). doi:10.1007/978-90-481-9751-4

17. Pribil, M., Labs, M. \& Leister, D. Structure and dynamics of thylakoids in land plants. $J$. Exp. Bot. 65, 1955-1972 (2014). 
18. Kirchhoff, H. Structural changes of the thylakoid membrane network induced by high light stress in plant chloroplasts. Philos. Trans. R. Soc. Lond. B. Biol. Sci. 369, 20130225 (2014).

19. Pedersen, J., Xiao, S. \& Mortensen, N. A. Slow-light enhanced absorption for biochemical sensing applications: Potential of low-contrast lossy materials. J. Eur. Opt. Soc. 3, (2008).

20. Nevo, R., Charuvi, D., Tsabari, O. \& Reich, Z. Composition, architecture and dynamics of the photosynthetic apparatus in higher plants. Plant J. 70, 157-176 (2012).

21. Murchie, E. H. \& Lawson, T. Chlorophyll fluorescence analysis: a guide to good practice and understanding some new applications. J. Exp. Bot. 64, 3983-3998 (2013).

22. Lawson, T., Oxborough, K., Morison, J. I. L. \& Baker, N. R. Responses of photosynthetic electron transport in stomatal guard cells and mesophyll cells in intact leaves to light, CO2, and humidity. Plant Physiol. 128, 52-62 (2002).

23. Lawson, T., Oxborough, K., Morison, J. I. L. \& Baker, N. R. The responses of guard and mesophyll cell photosynthesis to $\mathrm{CO} 2, \mathrm{O} 2$, light, and water stress in a range of species are similar. J. Exp. Bot. 54, 1743-1752 (2003).

24. Kouřil, R., Wientjes, E., Bultema, J. B., Croce, R. \& Boekema, E. J. High-light vs. lowlight: Effect of light acclimation on photosystem II composition and organization in Arabidopsis thaliana. Biochim. Biophys. Acta-Bioenerg. 1827, 411-419 (2013).

25. Wientjes, E., Van Amerongen, H. \& Croce, R. Quantum yield of charge separation in photosystem II: Functional effect of changes in the antenna size upon light acclimation. $J$. Phys. Chem. B 117, 11200-11208 (2013).

26. Lawson, T., Oxborough, K., Morison, J. I. L. \& Baker, N. R. Responses of photosynthetic electron transport in stomatal guard cells and mesophyll cells in intact leaves to light, CO2, and humidity. Plant Physiol. 128, 52-62 (2002).

27. Lopez-Garcia, M. et al. Enhancement and directionality of spontaneous emission in hybrid self-assembled photonic-plasmonic crystals. Small 6, 1757-1761 (2010).

28. Yeh, P., Yariv, A., Hong, C. \& Introduction, I. Electromagnetic propagation in periodic stratified media. I. General theory. J. Opt. Soc. Am. 91125, 423-438 (1977).

29. Kirchhoff, H. et al. Dynamic control of protein diffusion within the granal thylakoid lumen. Proc. Natl. Acad. Sci. 108, 20248-20253 (2011).

30. Lumerical Solutions, Inc. http://www.lumerical.com/tcad-products/fdtd/. Version 8.15.736. (2015). 
Figure 1| Blue leaf iridescence and iridoplasts in Begonia. a, Photograph of a leaf of Begonia grandis $x$ pavonina $(G x P)$. b, microscopy image showing iridoplasts in an epidermal cell under bright field epi-illumination. c, cryo-SEM image of a single iridoplast in Begonia GxP. d, typical reflectance spectrum measured at normal incidence for a single iridoplast (inset). Colour bar indicates real colors.

Figure 2 | Optical properties and modelling of iridoplast structure. a, TEM image of iridoplast (scale bar $1 \mu \mathrm{m}$ ) and inset of ultrastructure. Sketches show the parameters used in the optical model to define the photonic structure. b, experimental reflectance spectra normalized to maximum (absolute values available in Supplementary Fig.7) for two GxP iridoplasts. Solid and dotted red lines are calculated reflectance for $d_{\mathrm{s}}=125 \mathrm{~nm}$ and $d_{\mathrm{s}}=115 \mathrm{~nm}$ respectively with $N=8, N_{m}=3, M=9 \mathrm{~nm}$ and $L=7.5 \mathrm{~nm}$. c, iridoplast reflectance spectra as a function of the spacing between grana $\left(d_{\mathrm{s}}\right)$. Dashed line is $d_{\mathrm{s}}=125 \mathrm{~nm}$

Figure 3 | Enhanced absorption at reflectance sideband. a, calculation of $\gamma$ at normal incidence for the iridoplasts in Fig 2b. Shadow area shows integration area for the calculation of $\gamma_{\text {tot }}=5.6$ (7.6) nm with $d_{\mathrm{s}}$ $=125(115) \mathrm{nm}$ (see Methods). Bottom figure shows PAR spectra ( $\mathrm{q}_{\text {norm }}$ not at scale) under direct sun (yellow) and forest canopy shade (green $)^{6} . \mathbf{b}$, Electric field intensity within the iridoplast for wavelengths of interest shown as dashed lines in a) and $d_{s}=125 \mathrm{~nm}$. Light (dark) grey regions represent stroma (grana) and y position within the structure. $\mathbf{c}, \gamma$ as a function of incident angle $(\theta)$ and wavelength for $d_{\mathrm{s}}=125 \mathrm{~nm}$ and unpolarised incident light.

Figure 4 | Chlorophyll fluorescence images and quantum yield of Begonia plastids. a, chlorophyll fluorescence images of mesophyll chloroplasts (top) and iridoplasts (bottom). b, Maximum quantum yield of photosystem II (Fv/Fm) for guard cell chloroplasts, iridoplasts and mesophyll chloroplasts. Error bar shows standard deviation of the measurements. 